\title{
Dominance of Dispersion Interactions and Entropy over Electrostatics in Determining the Wettability and Friction of Two-Dimensional $\mathrm{MoS}_{2}$ Surfaces
}

Ananth Govind Rajan ${ }^{1}$, Vishnu Sresht ${ }^{1}$, Agilio A. H. Pádua ${ }^{2}$, Michael S. Strano ${ }^{1}$, and Daniel Blankschtein ${ }^{1 *}$

${ }^{1}$ Department of Chemical Engineering, Massachusetts Institute of Technology, Cambridge, Massachusetts 02139, United States

${ }^{2}$ Institut de Chimie de Clermont-Ferrand, Université Blaise Pascal and CNRS, 63171 Aubière, France

Corresponding Author

* Email: dblank@mit.edu

\section{Supporting Information Contents}

S1. Force-Field Parameters for $\mathrm{MoS}_{2}$

S2. Comparison of the Direct and Reciprocal Lattice Summations to Obtain the Electrostatic Potential and the Dispersion Interaction Energy above a Monolayer $\mathrm{MoS}_{2}$ Surface 3

S3. Obtaining the Axial-Radial Droplet Profile from MD Simulations ..................................... 6

S4. Water Contact Angle on Monolayer $\mathrm{MoS}_{2}$ Using Cylindrical Droplets ............................... 7

S5. Histograms for Free Energy Calculations using Bennett's Acceptance Ratio Method to

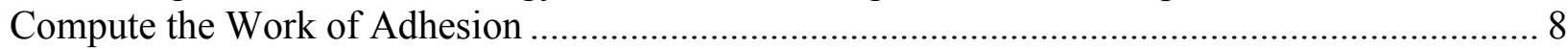

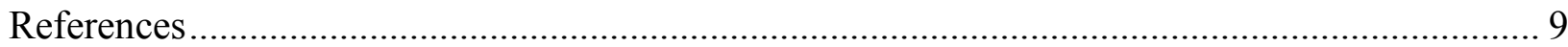




\section{S1. Force-Field Parameters for $\mathrm{MoS}_{2}$}

The force-field parameters utilized in our molecular dynamics (MD) simulations are listed in Table S1-1 below. Note that these parameters were validated against experimental contact angle data for bulk $\mathrm{MoS}_{2}$, and the experimental ranking of various solvents utilized in liquid-phase exfoliation of $\mathrm{MoS}_{2}$ sheets.

Table S1-1. Force-field parameters for molybdenum disulfide $\left(\mathrm{MoS}_{2}\right){ }^{1}$

\begin{tabular}{|c|c|c|c|}
\hline \multicolumn{4}{|c|}{ Non-Bonded Parameters } \\
\hline Atom & $\boldsymbol{\sigma}(\AA)$ & $\left(\frac{\mathrm{kJ}}{\mathrm{mol}}\right)$ & $q(\mathbf{e})$ \\
\hline Мo & 4.43 & 0.485 & +0.50 \\
\hline $\mathbf{S}$ & 3.34 & 2.085 & -0.25 \\
\hline \multicolumn{4}{|c|}{ Bonded Parameters } \\
\hline Type & \multicolumn{3}{|c|}{ Functional Form \& Parameters } \\
\hline $\begin{array}{c}\text { Harmonic Bond } \\
\text { (Mo-S) }\end{array}$ & \multicolumn{3}{|c|}{$U=\frac{1}{2} k_{b}\left(l-l_{b}\right)^{2}$} \\
\hline $\begin{array}{c}\text { Harmonic Angle } \\
\text { (S-Mo-S) }\end{array}$ & \multicolumn{3}{|c|}{$U=\frac{1}{2} k_{a}\left(\theta-\theta_{a}\right)^{2}$} \\
\hline $\begin{array}{c}\text { Harmonic Angle } \\
\text { (Mo-S-Mo) }\end{array}$ & \multicolumn{3}{|c|}{$U=\frac{1}{2} k_{a}\left(\theta-\theta_{a}\right)^{2}$} \\
\hline
\end{tabular}




\section{S2. Comparison of the Direct and Reciprocal Lattice Summations to Obtain the Electrostatic Potential and the Dispersion Interaction Energy above a Monolayer $\mathrm{MoS}_{2}$ Surface}

The electrostatic potential at points above a monolayer $\mathrm{MoS}_{2}$ sheet can be computed either using a direct-lattice summation (corresponding to all the point charges in the lattice), or using a reciprocal-lattice simulation, as indicated in the main text. For points close to the solid surface, a direct summation is adequate. On the other hand, even though for points far away from the surface, the direct summation converges slowly, the Ewald-summation technique can be used to derive a rapidly convergent series. ${ }^{2}$ However, the resulting mathematical expressions, following a tedious derivation, are convoluted..$^{2}$ Instead, we have utilized the summation in reciprocal space, as embodied in Eqs. (1) and (2) of the main text. Note that, for the SPC/E water model, $q_{O}=-0.8476 \mathbf{e}, \varepsilon_{O}=0.3166 \mathrm{~nm}, \sigma_{O}=0.650194 \frac{\mathrm{kJ}}{\mathrm{mol}}$, and $\varepsilon_{H}=\sigma_{H}=0 .^{3}$

Here, we present results for the electrostatic potential directly above an S-atom of the $\mathrm{MoS}_{2}$ sheet, using both direct- (Table S2-1) and reciprocal- (Table S2-2) space summations, for points at various distances: (i) closer to the sheet $(0.01 \AA, 0.05 \AA$, and $0.10 \AA$ ), and (ii) farther away from the sheet $(1.00 \AA, 2.00 \AA$, and $3.00 \AA$ ), the distance being measured from the top sulfur layer.

Table S2-1. Electrostatic potential at points directly above an S-atom on the $\mathrm{MoS}_{2}$ sheet, computed using a direct-lattice summation, at various distances from the sheet, using different lattice sizes. All the reported values are in Volts.

\begin{tabular}{|l|r|r|r|r|r|r|}
\hline \begin{tabular}{l} 
Distance above \\
$\begin{array}{l}\mathbf{M}_{2}(\AA) \\
\text { Lattice Size } \downarrow\end{array}$ \\
\hline $\mathbf{2 0 0} \times \mathbf{2 0 0}$
\end{tabular} & -355.389 & -67.510 & -31.624 & -0.8627 & -0.1037 & -0.0379 \\
\hline $\mathbf{4 0 0} \times \mathbf{4 0 0}$ & -355.374 & -67.495 & -31.609 & -0.8474 & -0.0884 & -0.0226 \\
\hline $\mathbf{1 0 0 0} \times \mathbf{1 0 0 0}$ & -355.364 & -67.486 & -31.600 & -0.8381 & -0.0792 & -0.0134 \\
\hline $\mathbf{2 0 0 0} \times \mathbf{2 0 0 0}$ & -355.361 & -67.483 & -31.596 & -0.8351 & -0.0761 & -0.0103 \\
\hline $\mathbf{4 0 0 0} \times \mathbf{4 0 0 0}$ & -355.360 & -67.481 & -31.595 & -0.8335 & -0.0746 & -0.0087 \\
\hline
\end{tabular}


Table S2-2. Electrostatic potential at points directly above an S-atom on the $\mathrm{MoS}_{2}$ sheet, computed using a reciprocal-lattice summation (Eq. (1) of the main text, without the factor of $q_{O}$ on the right hand side), at various distances from the sheet, using different lattice sizes. All the reported values are in Volts.

\begin{tabular}{|l|r|r|r|r|r|r|}
\hline $\begin{array}{l}\text { Distance above } \\
\begin{array}{l}\text { MoS } \\
\text { Lattice Size } \downarrow\end{array}\end{array}$ \\
\hline $\mathbf{5 0} \times \mathbf{5 0}$ & -156.854 & -63.416 & -31.461 & -0.8320 & -0.0730 & -0.0072 \\
\hline $\mathbf{1 0 0} \times \mathbf{1 0 0}$ & -243.263 & -67.200 & -31.593 & -0.8320 & -0.0730 & -0.0072 \\
\hline $\mathbf{2 0 0} \times \mathbf{2 0 0}$ & -318.632 & -67.478 & -31.593 & -0.8320 & -0.0730 & -0.0072 \\
\hline $\mathbf{5 0 0} \times \mathbf{5 0 0}$ & -353.897 & -67.480 & -31.593 & -0.8320 & -0.0730 & -0.0072 \\
\hline $\mathbf{1 0 0 0} \times \mathbf{1 0 0 0}$ & -355.350 & -67.480 & -31.593 & -0.8320 & -0.0730 & -0.0072 \\
\hline $\mathbf{2 0 0 0} \times \mathbf{2 0 0 0}$ & -355.358 & -67.480 & -31.593 & -0.8320 & -0.0730 & -0.0072 \\
\hline
\end{tabular}

As can be seen from the values reported in Tables S2-1 and S2-2, the direct-lattice summation converges using a small lattice size, for points very close to the surface (e.g., at a distance of $0.01 \AA$, there is only $0.008 \%$ discrepancy between using a $200 \times 200$ lattice versus a $4000 \times 4000$ lattice). On the other hand, for points farther away from the surface, the direct-lattice summation does not converge rapidly (e.g., at a distance of $3.00 \AA$, there is a $335 \%$ discrepancy between using a $200 \times 200$ lattice versus a $4000 \times 4000$ lattice). Conversely, the reciprocal-lattice summation converges very slowly at distances closer to the surface (e.g., using a $2000 \times 2000$ lattice for $0.01 \AA$ ), while it converges very rapidly for far away points (e.g., using just a $50 \times 50$ lattice for points at $2.00 \AA$ and $3.00 \AA$, which corresponds to $-25 \leq k_{1}, k_{2} \leq 25$ in Eq. (1) the main text). Therefore, at distances from the $\mathrm{MoS}_{2}$ sheet where most water molecules are present $(\sim 3 \AA)$, using the reciprocal-space summation to compute the electric potential is rapid and reliable.

Additionally, we present results for the dispersion interaction energy of a monolayer $\mathrm{MoS}_{2}$ sheet with a single O-atom located directly above an S-atom of the $\mathrm{MoS}_{2}$ sheet, using both 
direct- (Table S2-3) and reciprocal- (Table S2-4) space summations, for points at various distances: (i) closer to the sheet $(0.01 \AA, 0.05 \AA$, and $0.10 \AA$ ), and (ii) farther away from the sheet $(1.00 \AA, 2.00 \AA$, and $3.00 \AA$ ), the distance being measured from the top sulfur layer. As can be seen from the values reported in Table S2-3, in the case of dispersion interactions, due to their short-ranged nature, the direct-lattice summations converge using just a $200 \times 200$ lattice. The reciprocal-lattice summations converge slowly for smaller distances, and rapidly for larger distances (Table S2-4).

Table S2-3. Dispersion interaction energy of a single O-atom with a monolayer $\mathrm{MoS}_{2}$ sheet, at points directly above an S-atom on the $\mathrm{MoS}_{2}$ sheet, computed using a direct-lattice summation, at various distances from the sheet, using different lattice sizes. All the reported values are in $\mathrm{eV}$.

\begin{tabular}{|l|r|r|r|r|r|r|}
\hline \begin{tabular}{l} 
Distance above \\
$\begin{array}{l}\mathbf{M}_{2}(\AA) \\
\text { Lattice Size } \downarrow\end{array}$ \\
\hline $\mathbf{2 0 0} \times \mathbf{2 0 0}$
\end{tabular} & $6.75 \times 10^{28}$ & $2.76 \times 10^{20}$ & $6.75 \times 10^{16}$ & $6.74 \times 10^{4}$ & 15.448 & -0.0343 \\
\hline $\mathbf{4 0 0} \times \mathbf{4 0 0}$ & $6.75 \times 10^{28}$ & $2.76 \times 10^{20}$ & $6.75 \times 10^{16}$ & $6.74 \times 10^{4}$ & 15.448 & -0.0343 \\
\hline $\mathbf{1 0 0 0} \times \mathbf{1 0 0 0}$ & $6.75 \times 10^{28}$ & $2.76 \times 10^{20}$ & $6.75 \times 10^{16}$ & $6.74 \times 10^{4}$ & 15.448 & -0.0343 \\
\hline $\mathbf{2 0 0 0} \times \mathbf{2 0 0 0}$ & $6.75 \times 10^{28}$ & $2.76 \times 10^{20}$ & $6.75 \times 10^{16}$ & $6.74 \times 10^{4}$ & 15.448 & -0.0343 \\
\hline $\mathbf{4 0 0 0} \times \mathbf{4 0 0 0}$ & $6.75 \times 10^{28}$ & $2.76 \times 10^{20}$ & $6.75 \times 10^{16}$ & $6.74 \times 10^{4}$ & 15.448 & -0.0343 \\
\hline
\end{tabular}

Table S2-4. Dispersion interaction energy of a single O-atom with a monolayer $\mathrm{MoS}_{2}$ sheet, at points directly above an S-atom on the $\mathrm{MoS}_{2}$ sheet, computed using a reciprocal-lattice summation (Eq. (2) of the main text), at various distances from the sheet, using different lattice sizes. All the reported values are in $\mathrm{eV}$.

\begin{tabular}{|l|l|l|l|l|r|r|}
\hline $\begin{array}{l}\text { Distance above } \\
\mathbf{M}_{2}(\AA) \rightarrow \\
\text { Lattice Size } \downarrow\end{array}$ \\
\hline $\mathbf{5 0} \times \mathbf{5 0}$ & $1.26 \times 10^{27}$ & $9.65 \times 10^{19}$ & $5.18 \times 10^{16}$ & $6.74 \times 10^{4}$ & 15.448 & -0.0343 \\
\hline $\mathbf{1 0 0} \times \mathbf{1 0 0}$ & $4.74 \times 10^{27}$ & $2.10 \times 10^{20}$ & $6.66 \times 10^{16}$ & $6.74 \times 10^{4}$ & 15.448 & -0.0343 \\
\hline $\mathbf{2 0 0} \times \mathbf{2 0 0}$ & $1.62 \times 10^{28}$ & $2.73 \times 10^{20}$ & $6.75 \times 10^{16}$ & $6.74 \times 10^{4}$ & 15.448 & -0.0343 \\
\hline $\mathbf{5 0 0} \times \mathbf{5 0 0}$ & $5.11 \times 10^{28}$ & $2.76 \times 10^{20}$ & $6.75 \times 10^{16}$ & $6.74 \times 10^{4}$ & 15.448 & -0.0343 \\
\hline $\mathbf{1 0 0 0} \times \mathbf{1 0 0 0}$ & $6.65 \times 10^{28}$ & $2.76 \times 10^{20}$ & $6.75 \times 10^{16}$ & $6.74 \times 10^{4}$ & 15.448 & -0.0343 \\
\hline $\mathbf{2 0 0 0} \times \mathbf{2 0 0 0}$ & $6.75 \times 10^{28}$ & $2.76 \times 10^{20}$ & $6.75 \times 10^{16}$ & $6.74 \times 10^{4}$ & 15.448 & -0.0343 \\
\hline
\end{tabular}




\section{S3. Obtaining the Axial-Radial Droplet Profile from MD Simulations}
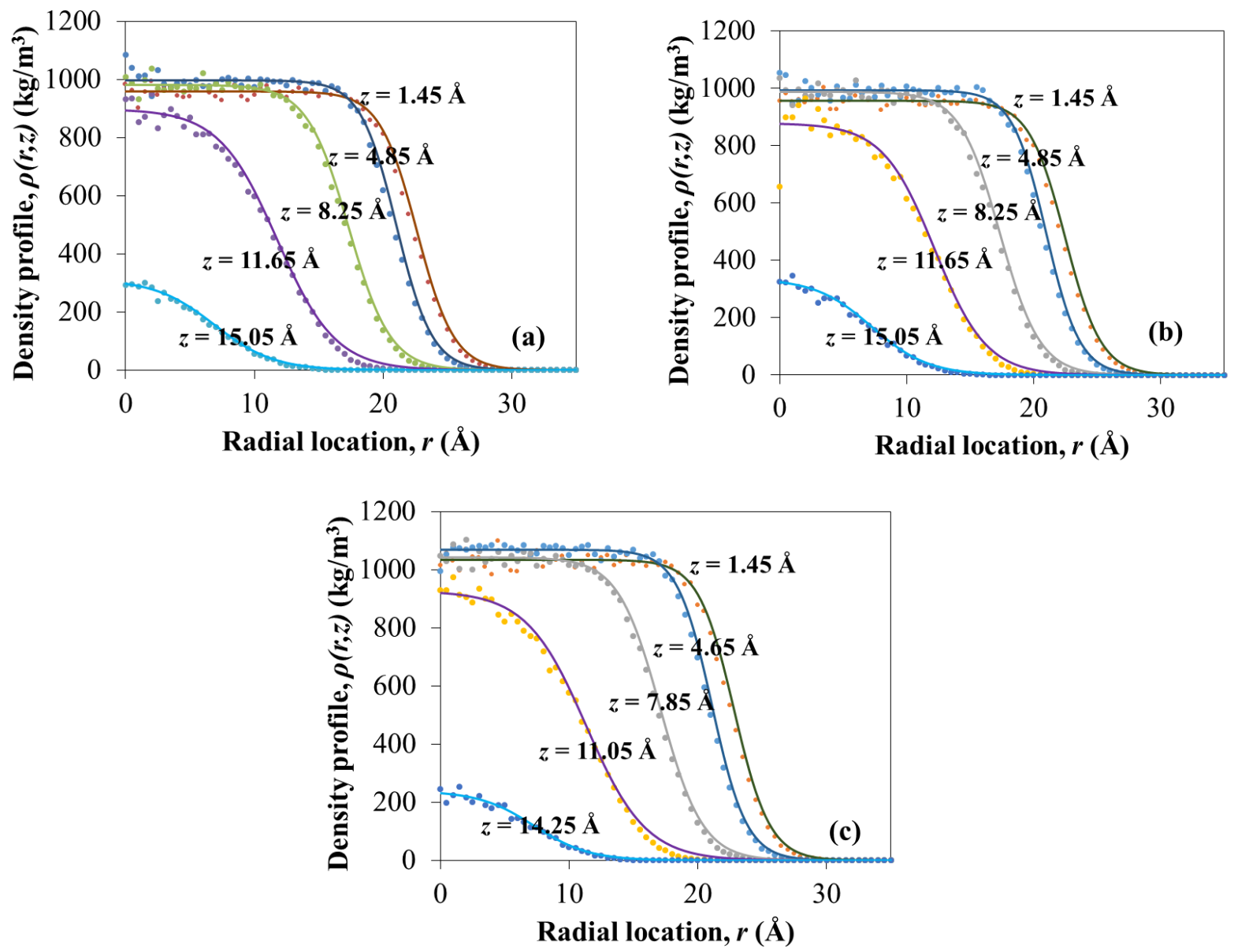

Figure S3-1. Radial density profile of water $\rho(r)$, in a droplet consisting of 500 water molecules, at various axial locations, $z$, above a monolayer $\mathrm{MoS}_{2}$ surface, in three different cases: (a) a frozen $\mathrm{MoS}_{2}$ sheet with polarized Mo and S atoms, (b) a frozen $\mathrm{MoS}_{2}$ sheet with nonpolar Mo and S atoms, and (c) a polarized, flexible $\mathrm{MoS}_{2}$ sheet. Note that the axial distance is measured from the top sulfur layer, and that these plots correspond to the three cases depicted in Figure 2 of the main text. The dots correspond to time-averaged MD simulations data, and the solid lines correspond to fits to Eq. (5) in the main text. 


\section{S4. Water Contact Angle on Monolayer $\mathrm{MoS}_{2}$ Using Cylindrical Droplets}

In order to compare the results of our contact angle simulations using hemispherical droplets with those obtained using cylindrical droplets, we also carried out MD simulations using cylindrical droplets. $N=1331,2635,5305$, and 10000 water molecules were separately simulated in a simulation box $\sim 5 \mathrm{~nm}$ wide. The simulations protocol and analysis procedure are described in the Methods section of the main text. The results obtained are summarized in Table S4-1. Although there should be no effect of the line tension in the case of cylindrical droplets, we see a variation in the contact angle as the droplet size increases, which is consistent with previous results. ${ }^{4,5}$ Note that the water contact angle obtained using the largest cylindrical droplet, consisting of 10000 water molecules, is $70.3^{\circ}$, which closely matches the contact angle of $71.5^{\circ}$ obtained using hemispherical droplets.

Table S4-1. Water contact angle on monolayer $\mathrm{MoS}_{2}$, obtained using cylindrical droplets consisting of $N$ water molecules.

\begin{tabular}{|l|r|}
\hline $\begin{array}{l}\text { \# of Water } \\
\text { Molecules in } \\
\text { Droplet, } \boldsymbol{N}\end{array}$ & $\begin{array}{r}\text { Contact } \\
\text { Angle }\end{array}$ \\
\hline $\mathbf{1 3 3 1}$ & $63.2^{\circ}$ \\
\hline $\mathbf{2 6 3 5}$ & $67.5^{\circ}$ \\
\hline $\mathbf{5 3 0 5}$ & $68.1^{\circ}$ \\
\hline $\mathbf{1 0 0 0 0}$ & $70.3^{\circ}$ \\
\hline
\end{tabular}




\section{S5. Histograms for Free-Energy Calculations using Bennett's Acceptance Ratio Method to Compute the Work of Adhesion}
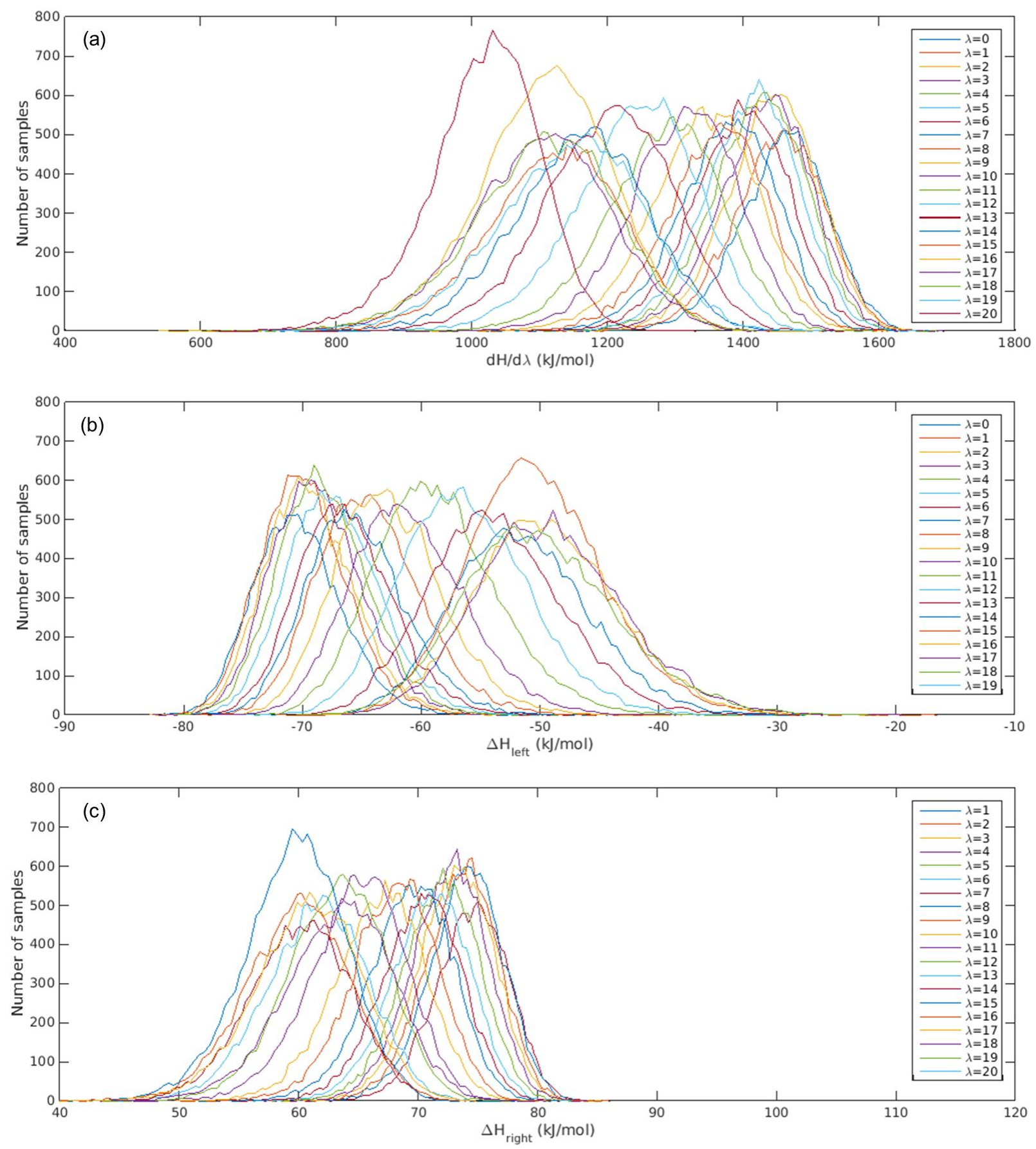

Figure S5-1. Histograms for the free-energy calculations to compute the work of adhesion of water on monolayer $\mathrm{MoS}_{2}$, depicting the number of samples at each computed value of: (a) $d H / d \lambda$, where $H$ is the Hamiltonian of the system, (b) $\Delta H$ for the left interval $(\lambda \rightarrow \lambda-\Delta \lambda)$, and (c) $\Delta H$ for the right interval $(\lambda \rightarrow \lambda+\Delta \lambda)$. It is evident that the intervals are overlapping sufficiently, implying that the sampling of the simulation is adequate. 


\section{References}

(1) Sresht, V.; Govind Rajan, A.; Bordes, E.; Strano, M. S.; Pádua, A. A. H.; Blankschtein, D. Quantitative Modeling of $\mathrm{MoS}_{2}$-Solvent Interfaces: Predicting Contact Angles and Exfoliation Performance using Molecular Dynamics, Submitted.

(2) Hod, O. Graphite and Hexagonal Boron-Nitride Have the Same Interlayer Distance. Why? J. Chem. Theory Comput. 2012, 8, 1360-1369.

(3) Berendsen, H. J. C.; Grigera, J. R.; Straatsma, T. P. The Missing Term in Effective Pair Potentials. J. Phys. Chem. 1987, 91, 6269-6271.

(4) Scocchi, G.; Sergi, D.; D’Angelo, C.; Ortona, A. Wetting and Contact-Line Effects for Spherical and Cylindrical Droplets on Graphene Layers: A Comparative MolecularDynamics Investigation. Phys. Rev. E 2011, 84, 061602.

(5) Rafiee, J.; Mi, X.; Gullapalli, H.; Thomas, A. V; Yavari, F.; Shi, Y.; Ajayan, P. M.; Koratkar, N. A. Wetting Transparency of Graphene. Nat. Mater. 2012, 11, 217-222. 